\title{
CAD/CAM for optomechatronics
}

\section{Haiguang Zhou, Min Han}

Haiguang Zhou, Min Han, "CAD/CAM for optomechatronics," Proc. SPIE 9663, Eighth International Topical Meeting on Education and Training in Optics and Photonics, 966329 (6 October 2003); doi: 10.1117/12.2208493

EDent: Eighth International Topical Meeting on Education and Training in Optics and Photonics, 2003, Tucson, Arizona, United States 


\title{
CAD/CAM for optomechatronics
}

\author{
Haiguang Zhou \\ Dept. of Physics, Xiamen University, Xiamen, Fujian, China \\ +86-592-2182412;.hgzhou@jingxian.xmu.edu.cn,hgzhou2001@yahoo.com.cn
}

\section{Min Han}

Dept. of Mechanical Engineering, Lujiang University, Xiamen, Fujian, China

\begin{abstract}
We focus at CAD/CAM for optomechatronics. We have developed a kind of $\mathrm{CAD} / \mathrm{CAM}$, which is not only for mechanics but also for optics and electronic. The software can be used for training and education. We introduce mechanical CAD, optical CAD and electrical CAD, we show how to draw a circuit diagram, mechanical diagram and luminous transmission diagram, from $2 \mathrm{D}$ drawing to $3 \mathrm{D}$ drawing. We introduce how to create $2 \mathrm{D}$ and $3 \mathrm{D}$ parts for optomechatronics, how to edit tool paths, how to select parameters for process, how to run the post processor, dynamic show the tool path and generate the $\mathrm{CNC}$ programming. We introduce the joint application of CAD\&CAM. We aim at how to match the requirement of optical, mechanical and electronics.
\end{abstract}

(C)2003 Optical Society of America

OCIS codes: (220.4880) Optomechanics 